\title{
Die „Kölnische Volkszeitung“ im Jahre 1932/33
}

\author{
von Heinz-Dietrich Fischer
}

Die am 1. April 1860 gegründete Zeitung „Kölnische Blätter“, die sich seit ihrem Entstehen als maßgebliches rheinisches Sprachrohr des politischen Katholizismus empfand $^{1}$ und 1869 den Titel „Kölnische Volkszeitung“ annahm, hatte mancherlei Repressalien zu erdulden, namentlich in der Zeit des Kulturkampfes ${ }^{2}$. Die Verlegerfamilie Bachem ${ }^{3}$ fand jedoch stets Mittel und Wege, um das Weitererscheinen des Blattes zu sichern. Trotz der aufstrebenden Berliner Konkurrenz des ZentrumsHauptblattes "Germania“ galt die "Kölnische Volkszeitung" zu Beginn des Ersten Weltkrieges als "die bedeutendste überregionale katholische Tageszeitung "4, doch betrug die Auflage 1917 nur rund 38000 tägliche Exemplare ${ }^{5}$.

Nachdern der Verlag "J. P. Bachem" schon 1920 zur "Kölnische Volkszeitung GmbH" erweitert worden war, wurde schließlich im April des Jahres 1926 die "Görreshaus

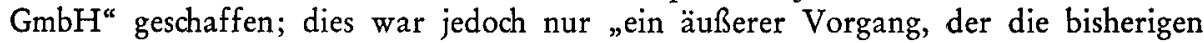

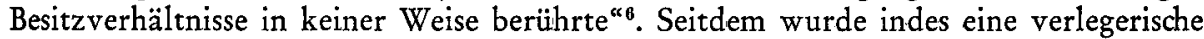
Expansion des Unternehmens deutlich, die in keiner Weise den publizistischen und finanziellen Möglichkeiten des Verlages entsprach. „Die geschäftliche Vorsicht und Redlichkeit des früheren Familienbetriebes“, so betont Rolf Kramer, „wich im Laufe der folgenden Jahre einem Wagemut, der einen allzu phantasievollen Optimismus zum Grundsatz machte. 1929 stellten sich die ersten finanziellen Schwierigkeiten ein. Am 11. September wurde das Unternehmen in eine AG umgewandelt", die „Görreshaus-Aktiengesellschaft " ${ }^{\text {"7 }}$.

Wie sich später herausstellte, war die Umwandlung des Verlages in eine AG vor allem wegen der inzwischen schwierigen Finanzlage des Unternehmens erfolgt. Neue Anteilseigner traten durch den Erwerb von Aktien in den Verlag ein, ohne zu ahnen, um welch wirtschaftlich ungesichertes Unternehmen es sich handelte. Als eine kreditgebende Bank im Sommer 1932 die Rückzahlung bestimmter Gelder verlangte, kam es zum Skandal, denn plötzlich wurde die Defizitsituation des Presseunternehmens der „Kölnischen Volkszeitung“ offenkundig. Der Verlagsleitung wurde in einem späteren Gerichtsverfahren u. a. vorgeworfen, fortgesetzten gemeinschaftlichen Betrug durch falsche Angaben über die tatsächliche ökonomische Basis des Blattes bei der Werbung von Aktionären begangen zu haben ${ }^{8}$.

Als dann noch Subventionen der Reichsregierung für die "Görreshaus AG“ bekannt wurden, meldete aus Köln die Agentur Telegraphen Union Ende Juli 1932:

„Weitere zwei Millionen Subventionen für die ,Kölnische Volkszeitung' festgestellt. Nachdem sich bereits vor kurzem herausgestellt, daß die ,Kölnische Volkszeitung zwei Millionen von der Reichsregierung [sic] Braun-Severing erhalten hatte, ist nunmehr - wie wir erfahren - festgestellt worden, daß diese Zeitung auf dem bekannten ,Umwege ${ }^{\prime}$ durch die Preußenkasse kurz vor der Entfernung der Regierung Braun-Severing aus dem Amte noch einmal zwei Millionen Mark erhalten hat."

Priv.-Doz. Dr. Heinz-Dietrich Fischer ist Wiss. Assistent und Lehrbeauftragter an der Sektion Publizistik und Kommunikation der Ruhr-Universität Bochum. In CS veröffentlichte er u.a. die Serie "CDU-nahe Lizenz-Zeitungen". 
Die nationalsozialistische Tageszeitung "Westdeutscher Beobachter" frohlockte hierauf?:

"Noch gestern hatte die ,Kölnische Volkszeitung' die Stirn zu behaupten, sie habe niemals von irgendeiner Seite finanzielle Zuwendungen erhalten. Die ... Meldung der vollkommen neutralen ,Telegraphen Union' straft die ,Kölnische Volkszeitung" Lügen! Nicht nur zwei Millionen, nein, vier Millionen Mark hat die ,Kölnische Volkszeitung c von der marxistischen Preußenregierung bekommen!! Gebt der Journaille die Antwort!“

Tags darauf wußte die gleiche Zeitung zu melden, daß das Defizit der „Kölnischen Volkszeitung" u. a. dadurch entstanden sein soll, daß leitende Redakteure überaus hohe Bezüge erhalten hatten, so beispielsweise der Chefredakteur ein Monatsgehalt von 1500 RM, sein Stellvertreter immerhin 1200 RM und einige weitere Redaktionsmitglieder noch mindestens $1000 \mathrm{RM}$ pro Monat ${ }^{10}$.

Im Herbst des Jahres 1932 wurden verschiedene Sanierungspläne für das Unternehmen diskutiert, die allesamt wiederum eine Beteiligung durch das Deutsche Reich vorsahen, welches sich jedoch angesichts der Wogen, die die Angelegenheit bereits aufgeworfen hatte, recht reserviert verhielt. Eine in Köln erscheinende Zeitung bemerkte dazu: „Man hatte ... daran gedacht, daß das Reich bei einer Wiedererhöhung des Kapitals durch die Dresdner Bank etwa zwei Millionen RM übernehmen würde. Dies scheint aber immerhin die Bedenken der Regierungsstellen gefunden zu haben ... Man ist also auf der Suche nach einer weniger offensichtlichen Methode, ein Unternehmen, ,das einfach nicht zusammenbrechen darf', auf Staatskosten zu erhalten..." "11.

Die gleiche Zeitung wußte dann Anfang Dezember 1932 zu melden, daß nun der Inhaber einer "bekannten süddeutschen Weckapparate-Fabrik" künftiger Majoritätsbesitzer der "Kölnischen Volkszeitung" sei, daß vorher jedoch "noch schwierige interne Veränderungen $\mathrm{zu}$ erwarten sein" würden, "die anscheinend nicht in der Richtung des bisherigen Kursus" der Zeitung lägen"

Politisch-publizistisch hatte die „Kölnische Volkszeitung“ stets als staatserhaltend und staatstragend gegolten, sie war ein Blatt der Mitte, des Maßes und des Ausgleiches. Doch nicht erst 1933, sondern bereits ein Jahr zuvor erfolgte im Zuge der verlagsinternen Umstrukturierungsmaßnahmen auch ein gewisser redaktioneller Umbruch, worüber Kramer berichtet ${ }^{13}$ :

„Es begann am 30. Mai 1932 mit dem Sturz Brünings, des letzten Zentrums-Kanzlers. Die , $\mathrm{KV}^{`}$ stand fortan in schärfster Opposition zu den Brüning-Nachfolgern. Es war das Ende einer Linie, die konsequent trotz aller Rückschläge der Weimarer Republik verfolgt worden war: das Eintreten für den Aufbau eines demokratischen Staatswesens. Die , $\mathrm{KV}^{\star}$ geriet in den folgenden Wochen immer mehr in Gegensatz zur Regierung Papen, den sie als ,Katastrophen-Kanzler' bezeichnete ${ }^{14}$. Am 6. Juli wurde die ,KV' für drei Tage verboten ${ }^{15}$. Das Urteil des Berliner Senats erregte in der deutschen Offentlichkeit großes Aufsehen, zumal der ,Vorwärts' kurz darauf ebenfalls verboten wurde. Der Kampf zwischen Zentrum und Nationalsozialismus verschärfte sich sprungartig, als Hitler in einer Rundfunkrede am 2. Februar 1933 das Zentrum als Novemberpartei ${ }^{`}$ bezeichnete, die zusammen mit den Sozialisten Deutschland in den vierzehn Weimarer Jahren ruiniert habe. Die Dolchstoß-Legende, jahrelang gegen die Sozialisten ins Feld geführt, verletzte die Partei und die ,KV aufs tiefste und machte sie zum erbitterten Feind des Nationalsozialismus ${ }^{16}$. Am 
18. Februar 1933 erfolgte dann der erste große Schlag der Nationalsozialisten gegen die Zentrumspresse. Die Regierung verbot grundsätzlich die Verbreitung von Aufrufen katholischer Verbände und Vereinigungen in den Zentrumszeitungen, da in diesen Appellen die Regierung beleidigt und verunglimpft werde. Bei Zuwiderhandlung wurde ein dreitägiges Verbot angedroht ${ }^{17}$. Am 19. Februar verfügte Göring nach einer persönlichen Unterredung mit dem ehemaligen Reichskanzler Marx die Aufhebung des angedrohten Verbots."

Je näher der Wahltermin vom 5. März 1933 rückte, desto mehr verschärften sich die nationalsozialistischen Repressalien gegen die "Kölnische Volkszeitung" die - zusammen mit einer Reihe anderer katholischer Blätter - bereits in der Zeit zwischen dem 19. und 21. Februar ein Erscheinungsverbot hinnehmen mußte ${ }^{18}$. Drei Tage vor der Wahl berichtete die Zeitung dann über eine Haussuchung in ihren Redaktionsräumen und die Beschlagnahmung von dort lagernden Plakaten der Zentrumspartei; abschließend hieß es in der täglichen Meinungsspalte "Die K. V. vermerkt" mit ironischem Ton: „Die K. V. vermerkt, daß sie nichts zu vermerken hat"19. Am Tag vor der Reichstagswahl schilderte die „Kölnische Volkszeitung" inzwischen neu aufgetretene Ereignisse detailliert ${ }^{20}$ :

„Die ,Kölnische Volkszeitung' war auf Grund der bekannten Verordnung für Samstag, Sonntag und Montag verboten und deswegen am Erscheinen verhindert. Wir gedachten also am Dienstagvormittag wieder eine Nummer herausgeben zu können. Dies wurde verhindert durch das folgende Ereignis:

Am Montagvormittag erschien eine Gruppe von uniformierten Nationalsozialisten in unserem Verlagshause und hißte vom Balkon des zweiten Stockwerks die Hakenkreuzflagge. Dann wurde ein stellvertretendes Vorstandsmitglied der GörreshausA.-G., ein Direktor der Kölner Verlagsanstalt und zwei Redakteure der , $\mathrm{KV}^{c}$ von den Nationalsozialisten festgenommen mit der Begründung, man müsse sie in Schutzhaft führen, um sie vor der Wut der Menge zu schützen. Dann wurden die vier Herren von den Nationalsozialisten zum Polizeipräsidium gebracht und dort von der Polizei in Schutzhaft genommen. Nachmittags wurden sie aus dieser Schutzhaft wieder entlassen.

Die im Görreshaus erschienenen Nationalsozialisten erklärten den Betrieb für besetzt und für drei Tage geschlossen. Alle Angestellten des Hauses mußten die Betriebsräume verlassen. Nur zwei Abteilungsleiter und einige Angestellte konnten zurück, bleiben. Der Betrieb blieb bis gegen $8 \mathrm{Uhr}$ abends besetzt und wurde erst um diese Zeit freigegeben.

Wir bitten die Leser um Entschuldigung, daß wir erst heute eine Möglichkeit besitzen, sie davon zu benachrichtigen, daß wir durch die obenerwähnten Vorfälle am Montag verhindert waren, für die rechtzeitige Herausgabe der Dienstagsausgabe Sorge zu tragen."

Der letzte Appell gegen den Terror der Nationalsozialisten erschien am Wahltag, dem 5. März 1933, er war überschrieben mit der Schlagzeile "Das steinerne Antlitz ${ }^{\text {«21. }}$. Mitte März, aus Anlaß der Wahlen zum preußischen Landtag, wurde die K. V. erneut für drei Tage verboten wegen des am 9. März erschienenen Artikels „Aschenbrödelrolle". Tags darauf erschien die Zeitung nur mit einem Blatt, welches diese Mitteilung des Kölner Polizeipräsidenten enthielt: „Im Namen des Herrn Oberpräsidenten der Rheinprovinz verbiete ich die ,Kölnische Volkszeitung', gemäß $\ 9$, Absatz 1, Ziffer 5 der Verordnung zum Schutze des deutschen Volkes vom 4. 2. 1933 
für die Zeit vom 11. bis einschließlich 13. März 1933“22. Und bereits am 11. März verkündete der Verlag der „Kölnischen Volkszeitung“, die Görreshaus AG, daß er sich aufzulösen beabsichtige ${ }^{\mathbf{2 3}}$. „Hier war der Anlaß nicht politisch“, bemerkte Koszyk, „sondern es ging um Geschäftsmethoden, die den Ruin des Unternehmens bewirkt hatten, aber doch politisch gegen das Zentrum umgemünzt wurden “24.

Kein geringerer als Karl Bachem, der Historiker des Zentrums und der katholischen Presse, ergriff im April 1933 dann als erster die Feder, um die sich für die Zeitung und für die Zentrumspartei abzeichnenden Zukunftsaussichten zu überdenken. Wegen der Bedeutung dieses bislang unpublizierten Dokumentes ${ }^{\mathbf{2 5}}$ mag es hier in voller Länge wiedergegeben sein:

„Wenn jetzt die Kölnische Volkszeitung reaktiviert werden soll, so fragt sich, welche Politik weiterhin von ihr getrieben werden soll oder kann.

Für die Fortführung der alten ,K. V. wäre zunächst der Name ,Neue Kölnische Volkszeitung' aufzunehmen, um einen deutlichen Schnitt zu machen gegen die Wirtschaft bei der bisherigen ,K. V. '. Die politische Haltung der alten K. V. war nach keiner Richtung hin bedenklich und braucht nicht desavouiert zu werden.

Weiterhin müßte die Sache der bestehenden, ruhmreichen alten Zentrumspartei aufrecht [!] und vertreten werden, soweit und solange es irgend geht. Es ist richtig, daß die bestehende Zentrumspartei belastet ist mit nur allzu vielen Vorkommnissen bei führenden Persönlichkeiten derselben, welche niemals hätten geduldet werden dürfen. Es ist auch richtig, daß namentlich bei der jüngeren Generation der Partei vielfach die mehr oder weniger unklare Stimmung herrscht, es müsse etwas ganz Neues geschaffen werden, um loszukommen von den Überlieferungen der alten Partei. Es ist endlich auch richtig, daß angesichts der neuen politischen Verhältnisse eine selbständige Politik in der Art, wie sie die Zentrumspartei bisher verfolgt hat, nicht mehr weiter getrieben werden kann. Es bleibt aber auch richtig, daß die bisherige Politik der Zentrumspartei, insbesondere der Zentrumsfraktion in Berlin im Großen Ganzen, der allgemeinen Richtung nach, richtig und einwandfrei war und, wenn man auch allerhand Einzelheiten kritisieren kann, den katholischen Prinzipien und Interessen in so tüchtiger und ehrenhafter Weise gedient hat, daß sie stets einen durchaus ehrenhaften Platz in der Geschichte des deutschen Volkes einnehmen wird.

Es ist sodann aber auch die Möglichkeit nicht aus dem Auge zu lassen, daß trotz allem es sich als unmöglich erweisen sollte, die Partei des Zentrums als solche aufrecht zu erhalten. Eine allgemeine selbstständige Politik ist der Zentrumspartei nicht mehr gestattet. Nichts, was irgendwie im Gegensatz zu der herrschenden nationalsozialistischen Partei steht, ist ihr erlaubt. Versammlungen sind nicht mehr zugelassen. Ihre Presse ist vollständig gefesselt. Das Vertrauen $\mathrm{zu}$ ihr ist bei weiten Kreisen untergraben. So könnte es sein, daß die äußere Organisation der Partei bald eintrodknet und dann langsam verfällt, gewissermaßen einschläft.

Was dann? Eine selbständige, staatspolitische Wirksamkeit ist, wie gesagt, für die Partei nicht mehr durchführbar. Es verbleibt aber noch die andere große Aufgabe, die kirchenpolitische Wirksamkeit, die Sorge für die spezifisch katholischen Grundsätze und Belange in unserer Zeit, die auch in der neu angehobenen nat. soz. Periode nicht entbehrt werden kann. Wenn die Partei in ihrer bisherigen Form nicht zu halten sein sollte, muß für diese Belange in irgend einer anderen Form gesorgt werden. Es muß dafür gesorgt werden, daß dann die Katholiken nicht einfach auseinander laufen und in Lethargie verfallen, was für viele nach den ungeheuren und nicht abreißenden 
Anregungen der letzten Zeit eine große Versuchung sein könnte. Das politische Ruhebedürfnis weiter Kreise ist ja erklärlich. Es darf aber nicht so weit kommen, daß dieses Ruhebedürfnis auch zu einem Erlahmen des spezifisch katholischen Gedankens führt.

Aber wie machen? Es ist daran gedacht worden, den Volksverein wieder zu beleben und diesem Bestreben nutzbar zu machen. Er sollte nach der Meinung mancher Kreise mit der Schulorganisation vereinigt und dann großzügig ausgebaut werden. Ob dieser Weg aussichtsreich ist, kann zweifelhaft sein. Auch der Volksverein ist in mancher Beziehung diskreditiert, weil von seinen finanziellen Schwierigkeiten so manches durchgesickert und infolge dieser Schwierigkeiten seine Tätigkeit in den letzten Jahren den nötigen großen Zug vermissen ließ. Dazu kommt, daß die allerdings etwas einseitige sozialpolitische Richtung des Volksvereins, welche ich für meinen Teil stets durchaus gebilligt habe, für manche sog. rechtsstehenden Kreise so wenig schmackhaft war, daß der Versuch einer Sammlung aller katholischen Richtungen in ihm wenig Aussicht haben dürfte.

Ein anderer Versuch dieser Art ist gemacht worden durch den Vice-Reichskanzler von Papen in Verbindung mit Emil Ritter durch die Gründung eines ,Bundes katholischer Deutscher Kreuz und Adler', welcher, den christlich-konservativen Gedanken im deutschen Volke vertiefen" soll, ,überparteilich" sein will, nicht ,an den Bestand von Parteien gebunden' zu sein erklärt und lediglich, durch sein Wirken diejenigen Parteien, welche sich dem christlich-konservativen Gedanken erschließen, zu befruchten' hofft. Ich halte diese Neugründung für sehr wenig zukunftsreich. Was ist in diesem Sinne der ,christlich-konservative Gedanke?" Man tut dem Gründer und manchen Mitgründern dieses recht losen Gebildes sicher kein großes Unrecht, wenn man annimmt, daß ihnen christlich-konservativ so ziemlich dasselbe ist wie christlich-reaktionär oder schließlich wie reaktionär ohne das Epitheton ornans christlich. Was damit getan sein soll gegenüber der immer schärfer hervortretenden Erscheinung, daß manche der führenden Persönlichkeiten des Nationalsozialismus für ihre Partei oder meinetwegen ihre Bewegung jetzt immer häufiger und immer heftiger den Charakter einer ,sozialistischen' Partei herauskehren, ist weniger verständlich. Auf keinen Fall hat diese Neugründung irgend etwas Anziehendes für die große Masse der katholischen Arbeiter, welche bisher treu zum alten Zentrum hielten.

Einen weiteren Versuch nach der Richtung der Sammlung der deutschen Katholiken hin wird wohl die Gründung eines ,Görresbundes' bedeuten, welcher am Gründonnerstag in Düsseldorf oder Köln steigen soll und an welcher Raederscheidt, Fürst Salm-Dyk, Nattermann beteiligt sein sollen. Diese Gründung soll, wie erzählt wird, die Billigung und Unterstuitzung des Kardinals Erzbischof Schulte haben. Was aus ihr wird, steht einstweilen dahin. Wenn es ihr gelänge, ein allgemeines Sammelbecken für alle Katholiken zu werden, welche politisch aktiv sind und im öffentlichen Leben für die katholischen Prinzipien und Interessen eintreten wollen, so wäre das allerdings eine große Sache. Vielleicht wird es richtig sein, zunächst mit allen Kräften diese Gründung zu unterstützen und den Versuch zu machen, sie nach allen Richtungen hin auszubauen, daß sie dem Bedürfnis genügt.

Wenn aber auch dieser Gedanke keinen allgemeinen Erfolg haben und nicht zu Großem führen sollte, was immerhin möglich ist, was dann?

Dann bleibt nichts als die Schaffung einer neuen großen katholischen Zeitung, welche, wenn sie schon auf selbständige Staatspolitik, insbesondere auf selbständige Sozial- 
politik verzichten muß, durch umso eifrigere Pflege des katholischen Gedankens die Katholiken geistig regsam und ihnen einen geistigen Mittelpunkt bietet. Der gegebene Ort dafür ist Köln, die wohl beste Form eine ,Neue Kölnische Volkszeitung‘.

Welche Politik könnte wohl eine solche Zeitung betreiben? Es ist klar, daß sie keinerlei Politik treiben kann, welche im Gegensatz steht zu dem heutigen nationalsozialistischen Regiment. Wenn sie das wollte, würde sie sofort völlig erschlagen werden, und eine Zeitung, welche nicht mehr besteht, kann nun einmal auch nichts mehr wirken. Zudem ist anzuerkennen, daß heute die nat. soz. Regierung die Obrigkeit ist, welche besteht', der gegenüber also der Katholik nach der klaren Vorschrift seiner Kirche die Untertanenpflicht zu leisten hat. Wie die Katholiken seit der Weimarer Verfassung das republikanische Regiment als die bestehende Obrigkeit anerkennen mußten und anerkannt haben, so müssen sie heute das nat. soz. Regiment als bestehende Obrigkeit anerkennen und ehren. Daran ist nicht vorbei zu kommen. Es dürfte also nichts gemacht werden, was den Verdacht erweckt, als ob man geradeaus oder hinten herum das bestehende nat. soz. Regiment wieder zu Fall bringen wollte. Wie früher das Zentrum und die deutschen Katholiken bis zum Letzten festgehalten haben an der Monarchie, wie sie dann seit 1919 ehrlich festgehalten haben an der damals aufgerichteten republikanischen Staatsform, nachdem diese festen Bestand angenommen hatte, so müssen sie heute aufrichtig mitmachen bei der neuen nat. soz. Staatsform. Diese ist zudem auf legalem Wege zustande gekommen, indem Reichspräsident von Hindenburg den Führer der nationalsozialistischen Bewegung, Adolf Hitler, mit dem Amte des Reichskanzlers betraute und dessen weitere Maßnahmen billigte oder zuließ.

Also zunächst nichts gegen das neue nat. soz. Regiment. Es ist aber auch manches in der heutigen nat. soz. Politik, was wir mit voller Uberzeugung und Hingabe mitmachen können und sollten.

Dahin rechne ich zunächst die Stärkung des nationalen Gesamtbewußtseins des deutschen Volkes, des Gefühles, daß dem Ausland gegenüber nur eine bedingungslose Einheit der Nation uns wieder zum Aufstieg bringen kann.

Sodann rechne ich dahin die Unterstützung der innerstaatlichen Vereinheitlichung, welche in den jüngsten Tagen so überraschend und auch so kraftvoll eingesetzt hat. Sie entspricht einfach dem alten großdeutschen Gedanken, wie er 1848 den gesamten deutschen Katholizismus beseelt und begeistert hat. Uberwindung des Partikularismus, ein einheitliches Reich, ein einheitlicher Reichswille, ohne die Möglichkeit partikularistischer Instanzen, der Reichsgewalt in den Arm zu fallen, - das ist zudem eine unbedingte Notwendigkeit, wenn wir wieder national und international die Formel vertreten: straffe Zusammenfassung unserer nationalen Kraft, sowohl dem Auslande gegenüber wie für die Gesundung unserer inneren Entwicklung. Der Kern dieser Formulierung ist die Beseitigung aller innerreichlichen Grenzen und Schranken, die Beseitigung des Unwesens der Einzelstaaten, kurz die Úberwindung des Partikularismus und die Pflege eines einheitlichen Reichsbewußtseins, welches endlich alle Stämme untereinander zur Überwindung ihrer besonderen Antipathien bringt und sie alle zur gleichberechtigten nationalen Mitarbeit herbeizieht - wie sie ja eben jetzt endlich vom Nationalsozialismus konsequent in Angriff genommen ist. Wenn dabei auch manche Rücksichtslosigkeiten unterlaufen, so können wir diese Politik doch geradlinig unterstützen.

Auch den Kampf des Nationalsozialismus gegen die Korruption in jeder Art können und sollen wir rückhaltlos unterstützen. Das wird auch zur inneren Gesundung unseres 
Zentrumsparteilebens führen, die dringend notwendig ist. Solche Fälle, wie wir sie jetzt erleben, von Höfle, Lange-Hegermann, Nientied, Hirtsiefer, Giesberts etc. bis zu der tollen Wirtschaft bei der Görreshaus A. G. dürfen nicht mehr vorkommen. Es hat gar keinen Sinn, das irgendetwas vertuschen oder beschönigen zu wollen. Die Luft muß gereinigt und dann in der Zukunft rücksichtslos rein erhalten werden.

Ganz dasselbe gilt von dem Kampf gegen Unsittlichkeit, gegen Schmutz und Schund, den wir alle Veranlassung haben, kräftig zu unterstützen. Wir haben diesen Kampf ja immer schon geführt. Er ist aber mit Rücksicht auf die törichte Einstellung der Sozialdemokraten und Demokraten in diesem Punkte und mit Rücksicht auf die Notwendigkeit des Koalitionsverhältnisses doch wohl allzu ,vorsichtig' geführt worden.

Dann aber kommen unsere besonderen katholischen Interessen. Nach der Rede Hitlers im Reichstag ist dafür eine ganz andere Möglichkeit, ein ganz anderer Boden geschaffen, als wir ihn bisher für möglich halten konnten. Die Freiheit der katholischen Kirche ist mit der Weimarer Verfassung endlich durchgesetzt. Sie zu erhalten ist jetzt die große Aufgabe, welche vielleicht eben soviel Arbeit und Mühe machen wird, wie früher die Aufgabe, sie zu erringen. Aber es wird vieler Vorsicht und Geschicklichkeit bedürfen, um hier nicht zurückgeworfen zu werden. Dazu gehört natürlich auch Aufrechterhaltung der Konkordate. Von einem Reichskonkordat haben wir kaum eine weitere Besserung zu erwarten. Es genügt uns die Aufrechterhaltung der bestehenden einzelstaatlichen Konkordate, wenn diese auch auf partikularistischer Grundlage aufgebaut sind. Man braucht in diesem Punkte die ,Gleichschaltung nicht zu übertreiben. Wenn aber die Reichsregierung ein Reichskonkordat anstrebt, haben wir keinen Grund, uns dem zu widersetzen.

Sodann weitere große Aufgaben:

Aufrechterhaltung der Gleichberechtigung der Katholiken bei Besetzung der Beamtenstellen.

Aufrechterhaltung des konfessionellen Schulwesens, ebenso wichtig: Aufrechterhaltung der ,Unterrichtsfreiheit', also der Zulassung unserer Privatschulen, vor allem der Ordensschulen.

Weiteres dazu später; namentlich über Sozialpolitik."

Die Bedenken und Befürchtungen Bachems sollten sich bestätigen, denn das faktische Ende der traditionsreichen „Kölnischen Volkszeitung" kam schnell: Am 9. April kündigte die Zeitung die Eröffnung des Liquidationsverfahrens an. Die letzte Nummer der K. V. in Köln wurde in der Nacht vom 29. auf den 30. April 1933 gedruckt. Die formelle Konkurserklärung der "Görreshaus AG" am 1. Mai und das baldige Verbot der Zentrumspartei erschütterten sowohl die wirtschaftliche Substanz als auch die geistige Widerstandskraft. Der Reichsabgeordnete Albert Hackelsberger, ein süddeutscher Großindustrieller erwarb aus der Konkursmasse das Verlagsrecht und gab die K. V. an den Essener Verlag Fredebeul \& Coenen in Druckauftrag, wo u. a. auch die "Essener Volkszeitung“" erschien. Schon am 2. Mai 1933 wurde die erste Nummer der neuen „Kölnischen Volkszeitung“ im Essener Verlag herausgegeben, zunächst im kleineren Format der „Essener Volkszeitung“, vom 4. Juni 1933 an, als der technische Apparat aus Köln in Essen installiert worden war, wieder im alten Gewande ${ }^{26}$.

Wenige Tage nach dieser Umstellung erschien in der „Kölnischen Volkszeitung" ein von Friedrich Muckermann SJ verfaßter Beitrag über die künftige Stellung der 
katholischen Presse im nationalsozialistischen Staat, der wegen seiner grundsätzlichen Ubberlegungen ein bedeutungsvolles Dokument darstellt ${ }^{27}$ :

„Die stürmisch voranschreitenden Ereignisse haben naturgemäß auch die katholische Presse in Mitleidenschaft gezogen. Diese Presse war mit den kirchlichen wie mit den staatlichen, mit den religiösen wie mit den politischen Interessen des deutschen Katholizismus innig verbunden. Sie war der Ausdruck des Empfindens des katholischen Volksteils, seiner Ideen, seiner Tradition, seiner schöpferischen Kräfte. In der Schicksalsgemeinschaft des deutschen Volkes webend, hat der katholische Volksteil doch immer seine besondere Sendung gehabt. Das gab seiner Presse jenen idealen Schwung, der namentlich in den Zeiten schwerer geistiger Bedrohungen christlichen und deutschen Wesens sich mächtig entfaltete. Es soll aus diesem gewaltigen Fragenkreis nur ein einziger Gesichtspunkt im folgenden hervorgehoben werden.

Jede Presse braucht Abonnenten. Wer in der Presse die wirtschaftlichen Vorbedingungen und Zusammenhänge nicht sieht, verkennt ihre Wesenheit. Dieser Abonnentenstand ist heute offensichtlich gefährdet. Es kommt das vor allem daher, daß zahlreiche Katholiken durch die Entwicklung der ständischen Zusammenfassungen aus ihrer gewohnten Bahn gerissen werden. Es ist zu natürlich, daß eine ständische Organisation ihre Mitglieder mit der in ihr herrschenden Atmosphäre vertraut machen will, und so wird zwangsläufig in den Berufsgemeinschaften eine eigene geistige Entwicklung einsetzen, die ihren Ausdruck u. a. auch in Presseorganen haben wird. Es könnten sich auf solche Weise leicht Ideengänge, die noch nicht hinreichend von uns geistig durchgearbeitet sind, über die an sich unpolitischen Berufsstände in den katholischen Volksteil hinein einen Weg suchen. Das könnte zu einer Zurückdrängung der katholischen Presse überhaupt führen, ohne daß irgend jemand das bewußt will.

Jeder Katholik wird verstehen, welche Gefahren diese Entwicklung mit sich bringt. $\mathrm{Da}$ das katholische Glaubensgut in Vereinigungen, die konfessionell gemischt sind, von selbst zurücktreten wird, da von einem Formwillen aus dem Ganzen der katholischen Idee heraus, dort nicht im vollen Sinne des Wortes die Rede sein kann, so wäre es leicht möglich, daß unsere Menschen von den eigensten und wichtigsten Lebenszentren abgedrängt werden. Es entsteht die Gefahr, daß nach und nach eine neue Trennung von Religion und Leben eintritt, ein Liberalismus, der heute genau die gleichen Folgen haben wird wie in früheren Zeiten. Die neuen Männer wollen diesen Liberalismus nicht. Sie bekämpfen ihn sogar. Adolf Hitler hat sich positiv zu einer inneren Verbindung von Kirche und Staat bekannt, aber in diesen Bereichen sind Zwangsläufigkeiten leicht stärker als ein noch so ehrlicher und tatkräftiger Wille führender Persönlichkeiten.

All diesen Gefahren gilt es zu begegnen. Unter allen Umständen ist es notwendig, daß die nationale Bewegung in innigster Verbindung mit den religiösen Kräften bleibe. Es werden ihr sonst jene Fundamente fehlen, auf denen allein eine starke Ideenentwicklung möglich ist. Es wird jener Schwung erlahmen, der aus dem religiösen Opfersinn erwächst. Es wird jene monumentale Größe dahinschwinden, die nur in der Verbindung mit den Tiefenkräften des menschlichen Herzens und der Geschichte unseres Volkes erreicht werden kann. Für den Katholizismus aber träte ein Sakristeichristentum in bedrohliche Nähe, daß [!] für die Formungen des öffentlichen Lebens keine Möglichkeit und bald auch keinen Sinn mehr hätte, ein ,Klerikalismus', der gerade dem echten Geistlichen zuwider ist. Es könnte eine Richtung entstehen, die in vollem Gegensatz stände zu der Geisteshaltung der großen päpstlichen Rundschreiben, die seit den Tagen Pius IX. sich mit größtem Nachdruck der Gestaltung 
des öffentlichen Lebens von der Religion her gewidmet haben, getreu den besten Traditionen des Abendlandes, dessen Charakteristik die Verbindung von Religion und Leben, von Kirche und Staat war. Wie entgehen wir nun praktisch all diesen Gefahren, und was kann die Presse insbesondere dazu tun?

Die deutschen Katholiken müssen sich bewußt werden, daß ihr Geistesgut und ihre Eigenart nur in einer starken katholischen Presse bewahrt werden können. Gerade jene von uns, die durch die ständische Gliederung den früheren stärker religiös betonten Gruppenbildungen entzogen werden, um nun dauernd in einer konfessionell gemischten ständischen Organisation zu wirken, werden die heilige Verpflichtung spüren, nun erst recht ganze katholische Menschen zu sein. Nur als ganze katholische Menschen können sie die katholischen Ideen der neuen Bewegung zuführen, nur mit dem Willen zu heiliger Unbedingtheit das Glaubensgut für sich und ihre Familie bewahren. Dazu bedürfen sie der Hilfe der katholischen Presse. Es muß zum Grundsatz werden, jetzt mehr noch als früher, daß jedes katholische Haus eine katholische Zeitung habe. Gerade die Gebildeten werden sich darüber klar werden müssen, daß nur dauernde Vertiefung in die katholische Geisteswelt sie für die Aufgaben von heute und morgen schulen kann.

Freilich muß dann aber auch die katholische Presse diesen neuen Bedürfnissen entsprechen. Es ist das für sie nicht so leicht, wie mancher Außenstehende glaubt. Wir können erst dann richtig über die Dinge schreiben, wenn wir sie seelisch gemeistert haben. Die Entwicklung geht rasend voran, gestattet kaum Besinnung. Sie hat das Tempo einer Revolution, nicht aber den ruhigen Rhythmus der Natur. Dennoch muß die Presse ihre Aufgaben erfüllen, denn ohne geistige Durchdringung werden die besten Bewegungen auf Irrwege geraten und die tiefsinnigsten Symbole ihren Sinn verlieren. Die katholische Presse wird mehr noch als früher aus dem eigensten katholischen Kulturgut schöpfen. Sie wird in allen ihren Sparten eine lebendige katholische Atmosphäre entwickeln. Sie wird den Wandel der Dinge vom Standpunkt der unwandelbaren Wahrheit aus betrachten und alle die Spannungen, die dauernd zwischen Zeit und Ewigkeit liegen, zum Ausdruck bringen. Sie wird das tun aus dem ehrlichen Willen heraus, die katholischen Kräfte der neuen Bewegung zur Verfügung zu stellen.

Sie wird es aber gleichermaßen tun aus der ebenso heiligen Verpflichtung heraus, wie früher für die volle soziale, wirtschaftliche und politische Gleichberechtigung der katholischen Minderheit in Deutschland sich einzusetzen. Es wäre schändlich von uns, wollten wir die Treue zum Neuen durch die Untreue am Alten erkaufen, und welche Charakterlosigkeit müßte am Ende einer Entwicklung stehen, die mit schändlichem Verrat ihren Anfang genommen hätte!

Es gibt ein Band, das die katholischen Volksgenossen zu einer eigenen Kulturgemeinde zusammenschließt. Die Möglichkeit, unser Leben nach katholischen Grundsätzen und Formen zu gestalten, ist uns gesichert durch das Hitlerwort von den Rechten und Freiheiten der Kirche, die anerkannt werden sollen. Unsere katholische Gemeinschaft gliedert sich selbstverständlich und natürlich ein in die Volksgemeinschaft. Sie ist die älteste Kulturgemeinschaft der christlichen Zeit auf deutschem Boden, in diesem Sinne die deutscheste Volkskirche und Volksgemeinschaft, die es gibt. Wenn Reichsminister Dr. Goebbels von einer Freiheit der Presse spricht, die nicht einer vollkommenen Uniformierung weichen soll, dann wird die Presse des katholischen Volksteils eine solche Freiheit zu allererst für sich in Anspruch nehmen dürfen, und wir erwarten die volle Anerkennung dieser Freiheit von dem kommenden Journalistengesetz. Der katholische Volksteil aber wird alles tun, um sich seine Presse zu erhalten. Kostet es 
Opfer, nun so fordern wir diese Opfer. Wir fordern sie mit dem gleichen Freimut wie die nationale Erneuerungsbewegung Opfer vom Volke fordern muß. Ist die ganze Nation eine Opfergemeinschaft, so ist es der katholische Volksteil noch in einem besonderen Sinne, steht doch in seiner Mitte der Opferaltar. Die Kraft, die uns daher kommt, wird uns in dieser Stunde nicht verlassen."

Die „Kölnische Volkszeitung “ führte nach ihrem Umzug nach Essen, der zeitlich mit der Auflösung der Zentrumspartei zusammenfiel, nur noch ein Schattendasein. Sie sank schnell zum bloßen Nachrichtenblatt herab, das die vom Wolffschen Telegraphenbüro, dem späteren Deutschen Nachrichtenbüro, verbreiteten offiziellen Nachrichten abdruckte, aber keine eigene Meinung mehr vertreten konnte. Von Januar 1937 an wurde das Format der KV auf $50 \times 38 \mathrm{~cm}$ verkleinert, seit Frühjahr 1940 erschien die Zeitung nur noch mit durchschnittlich vier Seiten. Als ihr Hauptschriftleiter, Max Horndasch, im Februar 1941, drei Monate vor Einstellung der Zeitung im Essener Gestapo-Gefängnis eine Woche inhaftiert wurde, erschien im Impressum hinter seinem Namen der Vermerk „verreist“. Die letzte Ausgabe der KV erschien am 31. Mai 1941. Es war die 150. Nummer des 82. Jahrgangs ${ }^{28}$.

\section{Anmerkungen:}

1. Vgl. zu ihrer frühen Entwicklungsgeschichte vor allem Karl Bachem: Josef Bachem - ein Altmeister der Presse, Köln 1938 (= Bd. 3 zu K. Bachem: Josef Bachem und die Entwicklung der katholischen Presse in Deutschland, 1912).

2. Vgl. Hans Joachim Reiber: Die katholische deutsche Tagespresse unter dem Einfluß des Kulturkampfes, phil. Diss. Leipzig 1930, Görlitz 1930.

3. Vgl. hierüber Georg Hölscher: 100 Jahre J. P. Bachem, Köln 1918.

4. Rolf Kramer: "Kölnische Volkszeitung“ (1860-1941), in: Heinz-Dietrich Fischer (Hrsg.): Deutsche Zeitungen des 17. bis 20. Jahrhunderts, München-Pullach 1972, S. 260.

5. Oskar Michel (Bearb.): Handbuch Deutscher Zeitungen 1917, Berlin 1917, S. 181.

6. Rolf Kramer, a. a. O., S. 263.

7. Daselbst, S. 264; vgl. auch Richard Lewinsohn (Morus): Das Geld in der Politik, Berlin 1930, S. 207; außerdem Kurt Koszyk: Deutsche Presse 1914-1945, Berlin 1972, S. 300 f.

8. Vgl. die Beschuldigungen insgesamt in: Der Görreshaus-Prozeß, in: ${ }_{n} \mathrm{Kölnische} \mathrm{Volkszei-}$ tung “, Nr. 208 (3. August 1933), S. 3. Weitere Hinweise auf zeitgenössische Informationen bei M. Schmolke: Die schlechte Presse, Münster 1971, S. 254 f.

9. Vgl. Die "Kölnische Volkszeitung" entlarvt, in: "Westdeutscher Beobachter" (Köln) vom 29. Juli 1932.

10. Vgl. Riesengehälter im Kölner Görreshaus!, in: „Westdeutscher Beobachter“ (Köln) vom 30. Juli 1932.

11. Kölner Görreshaus wird saniert, in: „Kölner Kurier“" vom 7. Oktober 1932.

12. Besitzwechsel bei Kölner Görreshaus, in: „Kölner Kurier" vom 2. Dezember 1932.

13. Rolf Kramer, a. a. O., S. 226.

14. Vgl. "Kölnische Volkszeitung" vom 26. Juli 1932.

15. Vgl. "Kölnische Volkszeitung" vom 7./9. Juli 1932.

16. Vgl. "Kölnische Volkszeitung" vom 5. Februar 1933.

17. Vgl. Erich Matthias/Rudolf Morsey (Hrsg.): Das Ende der Parteien 1933, Düsseldorf 1960.

18. Vgl. Karl Aloys Altmeyer: Katholische Presse unter NS-Diktatur, Berlin 1962, S. 17.

19. Vgl. „Kölnische Volkszeitung“ vom 2. März 1933.

20. Eine Erklärung, in: „Kölnische Volkszeitung", 74 Jg./Nr. 70 (4. März 1933), S. 1, Sp. 5.

21. Vgl. „Kölnische Volkszeitung“ vom 5. März 1933.

22. Verboten, in: „Kölnische Volkszeitung“ vom 10. März 1933.

23. Vgl. „Frankfurter Zeitung“, Nr. 623/624 vom 23. August 1933.

24. Kurt Koszyk: Das Ende des Rechtsstaates 1933/34 und die deutsche Presse, in: Emil Dovifat/Karl Bringmann (Hrsg.): Journalismus, Bd. 1, Düsseldorf 1960, S. 61. 
25. $\mathrm{C}$ [arl] B [achem]: Maschinenschriftliches Manuskript vom 11. April 1933, in: Historisches Archiv der Stadt Köln, Nachlaß Bachem, Abt. 1006, Nr. 856, 6 gez. Bl.

26. Rolf Kramer, a. a. O., S. 267.

27. Friedrich Muckermann: Die Stunde der katholischen Presse, in: „Kölnische Volkszeitung“ (Essen), 74. Jg/Nr. 119 (5. Mai 1933), S. 1, Sp. 3f.

28. Rolf Kramer, a. a. O., S. 267.

\section{S U M M A R Y}

With the exception of "Germania", published in Berlin, the "Kölnische Volkzeitung" was the leading daily of the Catholic Zentrum Party in the old German Kaiser-Reich and after World War I. In 1932 the publishers, "Görreshaus Aktion Gesellschaft" in Cologne faced an economic crisis. Thus weakened, the paper stood up against the National Socialist power take-over ("Machtergreifung"). The paper fought politically against the totalitarian demands of the Hitler party and at the same time it lost ground financially, and on the 1st May 1931 the publishing house went bankrupt. The paper was published the following day in Essen, (printed by Fredebeul and Coenen). It never again reached its earlier political importance. On the 5th July 1933 the German Zentrum Party suspended its own activities. A text from Karl Bachem, published here for the first time, regarding the possibility of a future "Neue Kölnische Volkzeitung", together with the deliberations of Friedrich Mudkermann on the importance of the Catholic Press under the Hitler "Reich" concludes this article.

\section{RESUME}

Le „Kölnische Volkszeitung“ était, outre le „Germania“ publié à Berlin, le plus important quotidien du parti centriste catholique de l'Empire allemand et de la République de Weimar. En 1932, sa maison d'édition, la "Görreshaus Aktien-Gesellschaft" de Cologne, affronta un crise économique. Ainsi, au printemps 1933, le journal se vit-il affaibli face à la prise du pouvoir par les nationaux socialistes. Le journal fut privé de ses moyens financiers au moment même où il devait mener la lutte politique contre les revendications totalitaires du régime hitlérien: le 1er mai 1933 suivit la mise en faillite de la maison d'édition „Görreshaus AG“. En vérité, le journal put, dès le 2 mai 1933, continuer de parâ̂tre à Essen (imprimé par Fredebeul \& Coenen). Il n'obtint cependant jamais plus l'importance politique qu'il avait atteinte pendant son apogée. Le 5 juillet 1933, le parti centriste allemand suspendit de luimême ses activités. Un texte de Karl Bachem, publié ici pour la première fois, concernant l'éventualité d'un "Neue K. V.“ à venir, de même que les réflexions de Friedrich Muckermann SJ sur l'importance de la presse catholique dans l'état nazi mettent un terme à l'illustration de cette année de crise.

\section{R E S U MEN}

El "Diario popular de Colonia“ (Kölnische Volkszeitung) era, junto al „Germania“ de Berlín, el periódico más destacado del Partido Centrista Alemán (católico) en la Alemania del segundo Reich y en la de la república de Weimar. En 1932 la casa editora del periódico, la "Görreshaus Aktien-Gesellschaft" de Colonia sufrió una crisis financiera. El rotativo seguía encontrandose en condiciones poco solventes cuando el Nacional Socialismo llegó al poder en la primavera de 1933. Mientras el diario luchaba políticamente contra las pretensiones totalitarias del regimen de Hitler, económicamente comenzó a naufragar: el l de Mayo de 1933 la editorial se declaró en suspensión de pagos. El periódico siguiô editándose ya desde el 2 de mayo del mismo año en Essen (impreso por la Fredebeul \& Coenen), pero ya no recuperó la importancia política de su mejor época. El 5 de Julio de 1933 se disolvió el Partido Centrista Alemán. Un texto de Karl Bachem sobre las posibilidades de un futuro „Nuevo Diario popular de Colonia" (Neue K. V.), publicado aquí ahora por primera vez, y consideraciones de Friedrich Muckermann SJ sobre la importancia de la prensa católica en el Estado Nacional Socialista perfilan la imagen de este año crítico. 